\title{
Understanding Filtration Efficiency Testing and Fit Testing in Filtering Facepiece Respirators (FFRs)
}

This fact sheet describes filtration efficiency testing and the proper fit testing procedures for a filtering facepiece respirator (FFR), explaining why both are necessary for your respirator to perform as expected. Your understanding of these procedures, along with information on how employers perform fit testing to minimize contaminant leakage into the respirator and determine how well it seals on your face, will enhance your knowledge of how an FFR helps protect your respiratory health.

\section{Testing Filtration Efficiency in FFRs}

The National Institute for Occupational Safety and Health $(\mathrm{NIOSH})$ tests and approves respirators that are used in occupational settings. The most well-known NIOSH-approved respirators are FFRs. FFRs are designed to cover areas of the user's face from the bridge of the nose to the chin. They are composed of a weave of electrostatically charged synthetic filter fibers (also called "filtration media") and elastic head straps. You wear FFRs over your nose and mouth to filter particles suspended in the air, called aerosols, as you inhale. FFRs provide protection against particles, but not gases or vapors, and therefore should not be used for respiratory protection against hazardous gases or vapors.

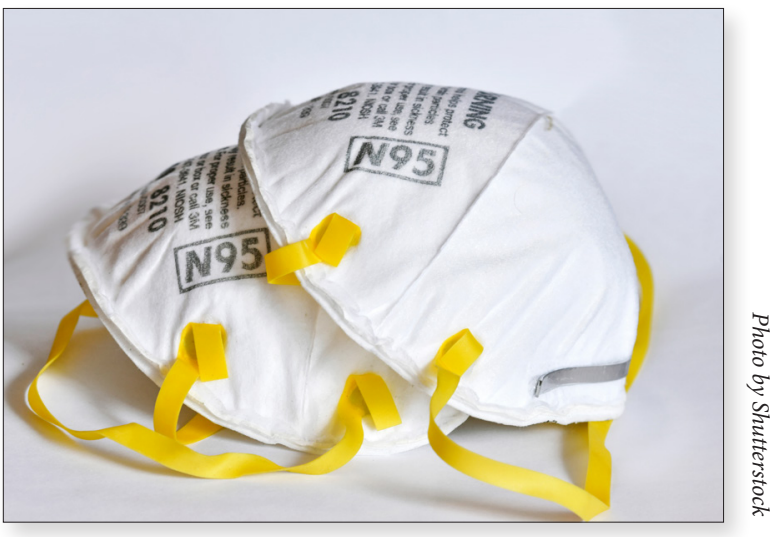

N95 filtering facepiece respirators.

There are nine filter classes for FFRs. The classes are made up of three levels of filtration efficiency $\left(95 \%, 99 \%\right.$, and $\left.99.97 \%{ }^{1}\right)$ and three series of protection against oil aerosols: (1) Not resistant to oil (N-type);

(2) Resistant to oil (R-type); and (3) oil Proof (P-type). These filtration efficiencies correspond to the percentage of the challenge aerosol collected by the FFR's filter media during testing. For example, an FFR marked N95 would indicate an N-series filter that is at least 95\% efficient. Because the most common FFR is an N95, in this fact sheet, N95 FFRs will be used as the example to demonstrate the concepts and procedures, but the discussion overall also applies to other FFR types.

\section{"Worst-case Scenario" Testing in FFRs}

All NIOSH-approved FFRs must pass a standardized test to verify that tiny particles like dust, dirt, viruses, and bacteria will be captured by the respirator's fibrous filtration media when worn. NIOSH tests simulate a "worst-case scenario" in relation to particle capture, which involves the two approaches discussed on the next page.

\footnotetext{
${ }^{1}$ When purchasing FFRs in these classes, a $99.97 \%$ filtration efficiency is shown as " 100 "-i.e., N100, R100, and P100 provide $99.97 \%$ filtration efficiency.
} 
Neutralizing the electrostatic charge of test particles. Because filters and particles may have opposite electrostatic charges, particles are naturally attracted to the filtration media and are bound there upon contact by electrostatic forces. NIOSH uses test particles without an electrostatic charge, which are harder to filter. This reduces the filter's efficiency, making it harder for the filter to trap particles that would otherwise be captured.

Testing particles that are challenging to filter. To measure particle penetration through the filtration media, NIOSH uses particle sizes known to be the most challenging to filter-particles with a 0.3 -micron diameter. ${ }^{2}$ These particles are too small to be seen without the aid of a microscope. By capturing 0.3-micron particles in standardized test conditions, NIOSH testing helps to ensure that FFRs will filter particles in the workplace at least as well as they do in the lab.

In the example used in this fact sheet for a NIOSH-approved N95 FFR, this two-pronged "worst-case scenario" approach provides a conservative measure of respirator efficiency that ensures the filtration media will capture at least $95 \%$ of non-oil aerosols when you inhale through the media.

\section{FFR Fit Testing by Employers}

Users of N95 FFRs need to understand that filtration efficiency alone does not guarantee protection. The respirator must also form a tight seal to the face. Without a tight seal, particles can leak into your breathing area. Therefore, fit testing is performed to minimize the amount of contaminant leakage into the respirator.

For employees whose job duties require them to wear respirators, the Occupational Safety and Health Administration (OSHA) requires that employers fit test these employees before the respirator is used in the workplace. Also, fit testing is required at least annually thereafter and whenever a different respirator facepiece is used or a change in the employee's physical condition could affect respirator fit. For this purpose, as detailed by OSHA guidelines, either qualitative or quantitative fit testing is used.

A qualitative fit test is a pass/fail test that determines whether a respirator user can detect (i.e., "taste") a test agent. In one common example of the procedure, after passing a taste threshold screening test without a respirator, the user dons (puts on) a respirator and test enclosure (which covers the head and shoulders). Next, the test agent is sprayed inside the enclosure to determine if its presence can be detected by the user. In this case, a "passing" test means that the user did not detect the taste, and therefore the make, model, and size of the test respirator could be assigned to that user.

When the test agent is generated, the user keeps the mouth slightly open with the tongue extended to ensure the ability to detect taste. The user also performs a series of simple exercises such as deep breathing and talking loudly and slowly enough to be heard by the tester.

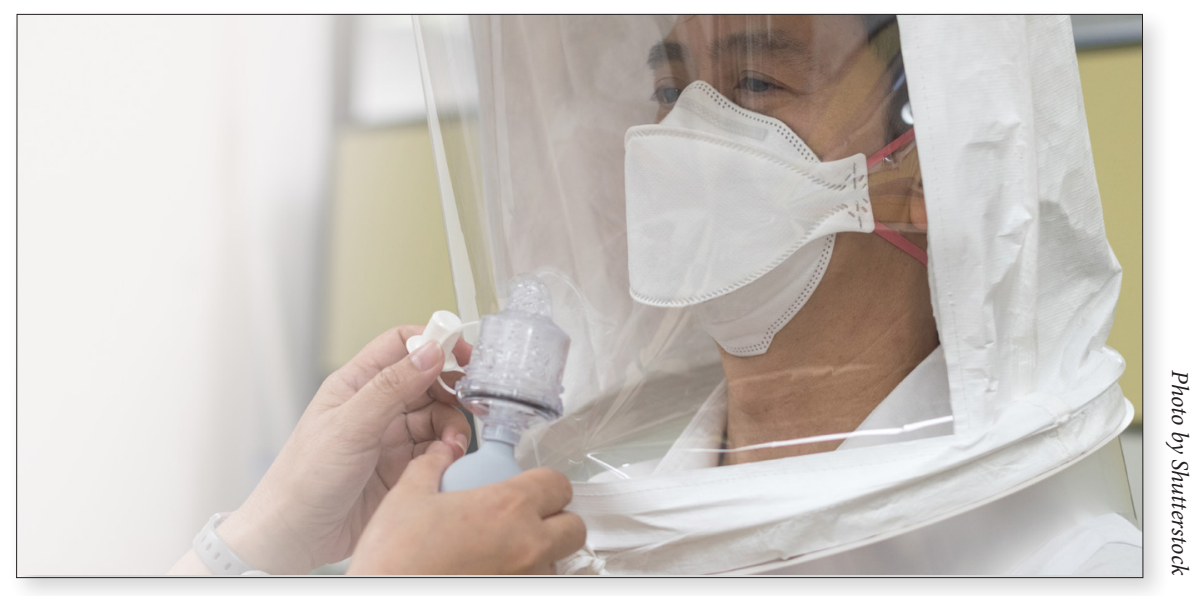

Participating in a qualitative fit test. 
A quantitative fit test uses an instrument to numerically measure inward leakage of the respirator that has been donned, allowing employers to calculate a "fit factor" for that respirator. A fit factor is a quantitative estimate of the fit of a particular respirator to a specific individual, typically expressed as the ratio of the concentration of a substance in ambient air to its concentration inside the respirator when worn.

During a quantitative fit test, users perform a series of simple exercises such as turning the head from side to side and bending over, and they read aloud from the "rainbow passage," which is a scripted paragraph representing a variety of sounds and mouth movements

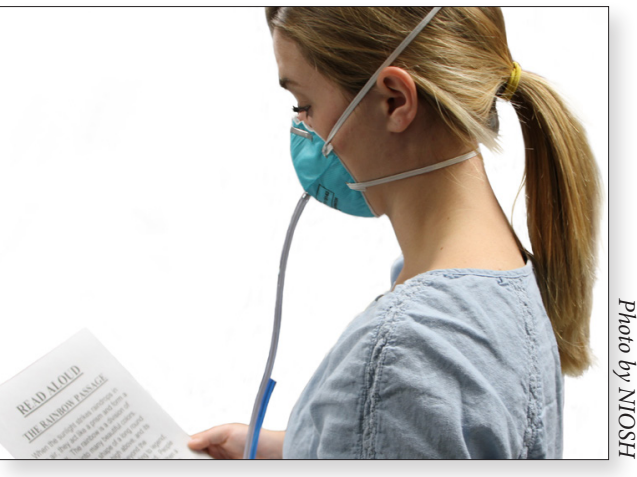

Reading the "rainbow passage" script aloud as part of a quantitative fit test. used in normal speech. These exercises help to ensure that the respirator seals well to the face even when the wearer is speaking or moving. ${ }^{3}$

Facial hair is a common reason for fit testing to fail because it can interfere with getting a tight face seal with the respirator and achieving maximum protection. Changes in facial characteristics like scarring, weight loss, or weight gain may also impact fit, and in such cases a different NIOSH-approved FFR than previously used may be needed.

\section{Ensuring That Your FFR Provides Protection}

$\square$ Get fit tested every year. Research shows that annual fit testing is necessary to account for changes in facial characteristics (e.g., from weight gain or loss) and to confirm that your chosen model of respirator fits you adequately.

$\square$ Review the user instructions for your FFR to confirm that you are doing a user seal check properly every time you don the respirator.

$\square$ Always wear your FFR where it provides adequate protection for the airborne contaminants in a particular area. Removing the respirator for even a short period of time could result in overexposure.

$\square$ Because an FFR is considered to be a disposable respirator, consult with the FFR manufacturer for specific guidance regarding its reuse and storage. For related information, you can also refer to NIOSH's end-of-service-life FAQs.

$\square$ Use NIOSH-approved FFRs for confidence in the stated filtration efficiency. Find lists of currently approved respirators on NIOSH's The Respiratory Protection Information Trusted Source and Certified Equipment List webpages.

\footnotetext{
${ }^{3}$ During a quantitative fit test, strain relief must be provided so the weight of the tube assembly does not artificially lower the fit factor by negatively affecting the seal of the respirator to the face. One way to provide such strain relief is by way of a lanyard securing the assembly to the wearer's clothing.
}

\section{Suggested Citation}

NIOSH [2021]. Understanding filtration efficiency testing and fit testing in filtering facepiece respirators (FFRs). By Schall J, Coffey C. Pittsburgh, PA: U.S. Department of Health and Human Services, Centers for Disease Control and Prevention, National Institute for Occupational Safety and Health. DHHS (NIOSH) Publication No. 2021-123 (revised 11/2021), https://doi.org/10.26616/NIOSHPUB2021123revised112021

DHHS (NIOSH) Publication No. 2021-123

(revised 11/2021)

DOI: https://doi.org/10.26616/

NIOSHPUB2021123revised112021

November 2021
Get More Information

Find NIOSH products and get answers to workplace safety and health questions:

1-800-CDC-INFO (1-800-232-4636) | TTY: 1-888-232-6348 CDC/NIOSH INFO: cdc.gov/info | cdc.gov/niosh

Monthly NIOSH eNews: $\underline{\text { cdc.gov/niosh/eNews }}$ 